\title{
ON THE ORBIFOLD EULER CHARACTERISTIC OF LOG DEL PEZZO SURFACES OF RANK ONE
}

\author{
DongSeon Hwang
}

\begin{abstract}
It is known that the orbifold Euler characteristic $e_{\text {orb }}(S)$ of a $\log$ del Pezzo surface $S$ of rank one satisfies the inequality $0 \leq e_{\text {orb }}(S) \leq 3$. In this note, we show that the orbifold Euler characteristic of $S$ is strictly positive, i.e., $0<e_{\text {orb }}(S)$. Moreover, we also show, by construction, the existence of log del Pezzo surfaces of rank one with arbitrarily small orbifold Euler characteristic.
\end{abstract}

\section{Introduction}

Throughout this paper, we work over the field $\mathbb{C}$ of complex numbers. A normal projective surface with quotient singularities is called a log del Pezzo surface if its anticanonical class is ample. A log del Pezzo surface is said to be of rank one if its Picard number is one. There have been extensive literatures devoted to the study of log del Pezzo surfaces motivated by questions in various areas of mathematics such as algebraic geometry, differential geometry and number theory. Many of the questions are reduced to the case of rank one.

Recall that the orbifold Euler characteristic $e_{\text {orb }}(S)$ of a normal projective surface $S$ with quotient singularities is defined as follows:

$$
e_{\text {orb }}(S)=e(S)-\sum_{p \in \operatorname{Sing}(S)}\left(1-\frac{1}{\left|G_{p}\right|}\right)
$$

where $G_{p}$ denotes the local fundamental group of the singular point $p$ of $S$.

From now on, we denote by $S$ a log del Pezzo surface of rank one. Then, the following bound on the orbifold Euler characteristic is known:

$$
0 \leq e_{\text {orb }}(S) \leq 3 \text {. }
$$

The upper bound immediately follows from the definition of the orbifold Euler characteristic since the Betti numbers of a $\log$ del Pezzo surface $S$ of rank

Received April 7, 2014.

2010 Mathematics Subject Classification. Primary 14J26, 14J17.

Key words and phrases. log del Pezzo surface of rank one, orbifold Euler characteristic.

This research was supported by Basic Science Research Program through the National Research Foundation of Korea(NRF) funded by the Ministry of Education(2011-0022904). 
one is the same as those of a complex projective plane. Note that $e_{\text {orb }}(S)=3$ if and only if $S$ is smooth, i.e., $S$ is a complex projective plane.

The lower bound was pointed out by Keel and McKernan ([7, Corollary $9.2]$ ). In the present paper, we show that the orbifold Euler characteristic of a log del Pezzo surface of rank one is strictly positive.

Theorem 1.1. The orbifold Euler characteristic $e_{\text {orb }}(S)$ of a log del Pezzo surface $S$ of rank one is positive. Hence,

$$
0<e_{\text {orb }}(S) \leq 3
$$

Note that, in Theorem 1.1, the surface $S$ is allowed to have only quotient singularities, i.e., log terminal singularities. The restriction on the singularities on $S$ is necessary. For instance, del Pezzo surfaces of rank one with exactly $5 \log$ canonical singularities are classified ([8, Theorem 1.1]). It turned out that each of those surfaces must have 4 ordinary double points and 1 rational non-klt log canonical singularity, hence $e_{\text {orb }}=0$.

In addition, the positivity of the anticanonical divisor $-K_{S}$ in Theorem 1.1 is necessary. An example of a normal projective surface $S$ with 5 quotient singularities such that $b_{2}(S)=1$ can be constructed by contracting 9 smooth rational curves forming the configuration $3 A_{1} \oplus 2 A_{3}$ on an Enriques surface ([4, Example 7.3]). It is easy to see that $e_{\text {orb }}(S)=0$.

Finally, we note that the above improved inequality is sharp in the sense that there exist log del Pezzo surfaces of rank one with arbitrarily small orbifold Euler characteristic.

Theorem 1.2. For each integer $m \geq 2$ and $n \geq 2$, there exists a log del Pezzo surface $S_{m, n}$ of rank one with exactly three singular points of type $D_{m+2}, D_{n+2}$, and $\frac{1}{m+n}(1,1)$, and $e_{\text {orb }}\left(S_{m, n}\right)=\frac{1}{m+n}+\frac{1}{4 m}+\frac{1}{4 n}$.

Here, the singularity of type $D_{n}$ refers to the singularity whose dual graph is the Dynkin diagram of type $D_{n}$ with each weight -2 . See Section 4 for the construction.

\section{Preliminaries}

\subsection{Notations}

Let $S$ be a normal surface with quotient singularities, and $f: S^{\prime} \rightarrow S$ be its minimal resolution. For each singular point $p$ of $S$, the dual graph of $f^{-1}(p)$ forms a tree, and the resolution graph is completely classified by Brieskorn ([2]). We will freely use his classification result and his notation. See also [4, Table 1] for the English translation. Also, we will sometimes denote the dual graph

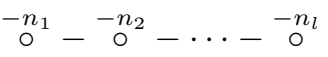


of the cyclic singularity by using the notation of the Hirzebruch-Jung continued fraction

We denote by

$$
\left[n_{1}, n_{2}, \ldots, n_{l}\right]=n_{1}-\frac{1}{n_{2}-\frac{1}{\ddots \cdot-\frac{1}{n_{l}}}}
$$

$$
\mathcal{F}:=f^{-1}(\operatorname{Sing}(S))
$$

the reduced exceptional divisor of $f$. A connected component $\mathcal{F}_{1}$ of $\mathcal{F}$ is called a linear chain if $\mathcal{F}_{1}$ is an exceptional divisor of a cyclic singularity, or it is called a fork if $\mathcal{F}_{1}$ is an exceptional divisor of a noncyclic singularity. An irreducible component $F_{1}$ of $\mathcal{F}$ is called a tip if it meets only one irreducible component of $\mathcal{F}-F_{1}$, or it is called a central component if it meets three irreducible component of $\mathcal{F}-F_{1}$.

\section{2. $\mathbb{Q}$-homology projective planes}

A normal projective surface $S$ with quotient singularities is called a $\mathbb{Q}$ homology projective plane if $b_{2}(S)=1$. Note that a log del Pezzo surface of rank one is nothing but a $\mathbb{Q}$-homology projective plane with ample anticanonical divisor. By the orbifold Bogomolov-Miyaoka-Yau inequality together with the observation by Keel and McKernan already mentioned in Section 1, we have the similar bound for the orbifold Euler characteristic.

Theorem 2.1. Let $S$ be a $\mathbb{Q}$-homology projective plane. Then,

$$
0 \leq e_{\text {orb }}(S) \leq 3
$$

Also, it is known that a $\mathbb{Q}$-homology projective plane admits at most 5 singular points.

Theorem $2.2([1,4])$. Let $S$ be a $\mathbb{Q}$-homology projective plane. Then, $S$ has at most 5 singular points, and $S$ has exactly 5 singular points if and only if $S$ is obtained by contracting 9 smooth rational curves forming the configuration $3 A_{1} \oplus 2 A_{3}$ on an Enriques surface.

The following special case of the theorem will play a crucial role in the proof of Theorem 1.1.

Corollary 2.3. Let $S$ be a $\mathbb{Q}$-homology projective plane. If $S$ is rational, then $S$ has at most 4 singular points. In particular, if $S$ is a log del Pezzo surface of rank one, then $S$ has at most 4 singular points.

\subsection{Log del Pezzo surfaces of rank one}

In this subsection, we review the classification theory of log del Pezzo surfaces of rank one initiated by Zhang ([12]), and further developed and reorganized by Gurjar and Zhang ([3]), Belousov ([1]), Kojima and Takahashi ([9], 
$[10])$, and Hwang and Keum([6]). We mainly follow the formulation in [6] for convenience.

Let $S$ be a $\log$ del Pezzo surface of rank one and $f: S^{\prime} \rightarrow S$ be its minimal resolution.

Lemma 2.4 ([12, Lemma 4.1]). Let $E$ be a $(-1)$-curve on $S^{\prime}$. Let $F_{1}, \ldots, F_{r}$ exhaust all irreducible components of $\mathcal{F}$ such that E. $F_{i}>0$. Suppose that $F_{1}^{2} \geq F_{2}^{2} \geq \cdots \geq F_{r}^{2}$. Then the $r$-tuple $\left(-F_{1}^{2}, \ldots,-F_{r}^{2}\right)$ is one of the following:

$$
(2, \ldots, 2, n), n \geq 2,(2, \ldots, 2,3,3),(2, \ldots, 2,3,4),(2, \ldots, 2,3,5) .
$$

An irreducible curve $C$ on $S^{\prime}$ is called a minimal curve if $C \cdot\left(-f^{*} K_{S}\right)$ attains the minimal positive value. From now on, we always assume that $C$ is a minimal curve.

Lemma 2.5 ([6, Lemma 3.3]). A minimal curve $C$ is a smooth rational curve.

We first analyze the case when $\left|C+\mathcal{F}+K_{S^{\prime}}\right| \neq \emptyset$.

Lemma 2.6. Let $C$ be a minimal curve. Suppose that $\left|C+\mathcal{F}+K_{S^{\prime}}\right| \neq \emptyset$. Then, $C^{2}=0$ or -1 , and if $S$ has exactly $n$ singular points, then $n-1$ of them are rational double points and the other singularity is either a cyclic singular point $p$ or again a rational double point. Moreover, if $\mathcal{F}^{\prime}$ is the reduced exceptional divisor of $f^{-1}(p)$, then one of the following holds:

(1) $\mathcal{F}^{\prime}$ consists of one irreducible component, which $C$ meets in a single point with multiplicity 2 or in two points,

(2) $\mathcal{F}^{\prime}$ consists of two irreducible components, whose intersection point $C$ passes through,

(3) $\mathcal{F}^{\prime}$ consists of at least two irreducible components, and $C$ meets the two end components of $\mathcal{F}$.

Proof. The assertion that $C^{2}=0$ or -1 follows from the first paragraph of Proof of Theorem 1.2 of [1]. The remaining part is exactly [6, Lemma 3.4].

Now, we analyze the case when $\left|C+\mathcal{F}+K_{S^{\prime}}\right|=\emptyset$.

Lemma 2.7 ([3, Proposition 3.6]). Let $C$ be a minimal curve. Suppose that $\left|C+\mathcal{F}+K_{S^{\prime}}\right|=\emptyset$. Then either $C$ is a $(-1)$-curve, $S \cong \mathbb{P}^{2}$, or $S$ is the Hirzebruch surface with the minimal section contracted. In particular, if $S$ has at least two singularities, then $C$ is a (-1)-curve.

From now on, we assume that the minimal curve $C$ is a $(-1)$-curve.

Lemma 2.8 ([1, Lemma 4.1]). Suppose that $S^{\prime}$ contains a minimal curve $C$ with $C^{2}=-1$. Suppose that $\left|C+\mathcal{F}+K_{S^{\prime}}\right|=\emptyset$. Then $C . \mathcal{F}^{\prime} \leq 1$ for any connected component $\mathcal{F}^{\prime}$ of $\mathcal{F}$.

It is well known that the number $C . \mathcal{F}$ is bounded. We give a proof for the convenience of readers.

Lemma 2.9. Suppose that $\left|C+\mathcal{F}+K_{S^{\prime}}\right|=\emptyset$. Then, $C . \mathcal{F}=1,2$ or 3 . 
Proof. By [12, Lemma 1.4], C.F $\geq 1$. By Lemma 2.8 and Corollary 2.3, C.F $\leq$ 4. Assume that $C . \mathcal{F}=4$. Then, $S$ has 4 singular points. By Lemma 2.4, $C$ meets two irreducible components $F_{1}$ and $F_{2}$ of $\mathcal{F}$ with $F_{1}^{2}=F_{2}^{2}=-2$. According to the classification of [10, Theorem 4.1], such a surface does not exist.

Assume that $C . \mathcal{F}=2$.

Lemma 2.10 ([12, Lemma 4.4]). Suppose that $S^{\prime}$ contains a minimal curve $C$ with $C^{2}=-1$. Suppose that $\left|C+\mathcal{F}+K_{S^{\prime}}\right|=\emptyset$, and that $C$ meets exactly two components $F_{1}, F_{2}$ of $\mathcal{F}$. Then either $F_{1}^{2}=-2$ or $F_{2}^{2}=-2$.

The following lemma is a special case of [10, Theorem 4.1].

Lemma 2.11. With the same assumption as in Lemma 2.10, assume further that $F_{1}^{2}=F_{2}^{2}=-2$. If $S$ contains 4 singular points, then the type of singularities is one the following two possibilities:

(1) $2 A_{1} \oplus[m] \oplus D_{m+2}$ for some integer $m \geq 3$,

(2) $3 A_{1} \oplus\langle m ; 2,1 ; 2,1 ; 2,1\rangle$.

Assume that $C \cdot \mathcal{F}=3$.

Lemma 2.12 ([3, Lemma 6.3, Lemma 6.6, Theorem 6.12 and Theorem 6.13]). Suppose that $S^{\prime}$ contains a minimal curve $C$ with $C^{2}=-1$. Suppose that $\left|C+\mathcal{F}+K_{S^{\prime}}\right|=\emptyset$, and that $C$ meets three components $F_{1}, F_{2}, F_{3}$ of $\mathcal{F}$. Denote by $\mathcal{F}_{i}$ the connected component of $\mathcal{F}$ containing $F_{i}$ for each $i=1,2,3$. Define

$$
G:=2 C+F_{1}+F_{2}+F_{3}+K_{S^{\prime}} .
$$

Then, either $G \sim 0$ or $G \sim \Gamma$ for some $(-1)$-curve $\Gamma$ such that $C . \Gamma=F_{i} . \Gamma=0$ for $i=1,2,3$. Furthermore, the following hold true.

(1) In the first case, for each $i=1,2,3, \mathcal{F}_{i}$ consists of only one connected component, i.e., $\mathcal{F}_{i}=F_{i}$, and $C$ meets no component of $\mathcal{F}-\left(F_{1}+F_{2}+\right.$ $\left.F_{3}\right)$.

(2) In the second case,

(a) $L=2-\left(F_{1}^{2}+F_{2}^{2}+F_{3}^{2}\right)$, where $L$ is the number of irreducible components of $\mathcal{F}$,

(b) each curve in $\mathcal{F}-F_{1}-F_{2}-F_{3}$ is a $(-2)$-curve or a $(-3)$-curve, and there are at most two $(-3)$-curves in $\mathcal{F}-F_{1}-F_{2}-F_{3}$,

(c) each connected component of $\mathcal{F}$ contains at most one $(-n)$-curve with $n \geq 3$,

(d) either there is another minimal curve $C^{\prime}$ such that $\left|C^{\prime}+\mathcal{F}+K_{S^{\prime}}\right| \neq$ $\emptyset$ or each $\mathcal{F}_{i}$ is a linear chain containing $F_{i}$ as a tip for each $i$.

\section{Proof of Theorem 1.1}

Let $S$ be a $\log$ del Pezzo surface of rank one with $e_{\text {orb }}(S)=0$ and $f: S^{\prime} \rightarrow S$ be its minimal resolution. We denote by $\mathcal{F}:=f^{-1}(\operatorname{Sing}(S))$ the reduced 
exceptional divisor of $f$. Let $L$ be the number of all irreducible components in $\mathcal{F}$.

By the definition of the orbifold Euler characteristic, we have

$$
\sum_{p \in \operatorname{Sing}(S)}\left(1-\frac{1}{\left|G_{p}\right|}\right)=3,
$$

where $G_{p}$ denotes the local fundamental group of the singular point $p$ of $S$. Thus, $S$ contains exactly four singular points by Corollary 2.3 , and we have the following list of all possible 4-tuples of orders of local fundamental groups:

- $(2,3,7,42),(2,3,8,24),(2,3,9,18),(2,3,10,15),(2,3,12,12)$, $(2,4,5,20),(2,4,6,12),(2,4,8,8),(2,5,5,10),(2,6,6,6)$, $(3,3,4,12),(3,3,6,6),(3,4,4,6),(4,4,4,4)$.

We call the above list by ORDERS. Elementary calculation provides the complete list of all possible singularity types corresponding to the given order of the local fundamental group.

TABLE 1. List of possible cyclic singularities

\begin{tabular}{|c|l|}
\hline Order & Types of cyclic singularities \\
\hline 2 & $A_{1}$ \\
3 & {$[3], A_{2}$} \\
4 & {$[4], A_{3}$} \\
5 & {$[5],[3,2], A_{4}$} \\
6 & {$[6], A_{5}$} \\
7 & {$[7],[4,2],[3,2,2], A_{6}$} \\
8 & {$[8],[3,3],[2,3,2], A_{7}$} \\
9 & {$[9],[5,2],\left[3,2^{3}\right], A_{8}$} \\
10 & {$[10],[4,2,2], A_{9}$} \\
12 & {$[12],[2,4,2],[3,2,3], A_{11}$} \\
15 & {$[15],[4,4],[8,2],[2,2,3,2,2],\left[3,2^{6}\right], A_{14}$} \\
18 & {$[18],[4,3,2],[3,3,2,2], A_{17}$} \\
20 & {$[20],[7,3],[2,6,2],\left[3,2^{3}, 3\right],\left[2,3,2^{5}\right], A_{19}$} \\
24 & {$[24],[5,5],[2,7,2],[4,2,4],[2,2,4,2,2],\left[3,2^{4}, 3\right],\left[2^{3}, 3,2^{3}\right], A_{23}$} \\
42 & {$[42],[2,6,2],[9,2,3],[2,2,6,2,2],\left[4,2^{3}, 4\right],\left[3,2^{3}, 3,2,2\right],\left[2,4,2^{7}\right], A_{41}$} \\
\hline
\end{tabular}

In Table 1, 'Order' means the order of the local fundamental group. In addition, we denote by $2^{a}$ the $a$-th iterates of 2 . For example, $\left[4,2^{3}, 4\right]$ means $[4,2,2,2,4]$.

If $S$ contains a noncyclic singularity, then the list is reduced to the following:

- $(2,3,8,24),(2,3,12,12),(2,4,5,20),(2,4,6,12),(2,4,8,8),(3,3,4,12)$,

since the order of the local fundamental group of a noncyclic singularity is divisible by 4 and at least 8 . 
TABLE 2. List of possible noncyclic singularities

\begin{tabular}{|c|l|}
\hline Order & Types of noncyclic singularities \\
\hline 8 & $D_{4}$ \\
12 & $D_{5}$ \\
20 & $D_{7}$ \\
24 & $D_{8}, E_{6},\langle 2 ; 2,1 ; 2,1 ; 3,1\rangle,\langle 3 ; 2,1 ; 2,1 ; 2,1\rangle$ \\
\hline
\end{tabular}

We call this reduced list by NONCYC. See Table 2 for the complete list of corresponding noncyclic singularities.

For a 4 -tuple $\left(q_{1}, q_{2}, q_{3}, q_{4}\right)$ on the list ORDERS, let $p_{i}$ be the singular point of $S$ whose local fundamental group is of order $q_{i}$, and let $\Delta_{i}$ be the reduced exceptional divisor of the minimal resolution of $p_{i}$.

Claim: $S$ admits at least one singular point that is not a rational double point.

Suppose that $S$ has at worst rational double points. Then, the anticanonical divisor $-K_{S^{\prime}}$ of the minimal resolution $S^{\prime}$ is nef and big, so the number of irreducible components of $\mathcal{F}$ is not greater than 8 which leads to a contradiction. On the other hand, log del Pezzo surfaces of rank one with only rational double points is completely classified ([11]). One can alternatively verify the assertion of Claim using this classification.

Let $C$ be a minimal curve on $S^{\prime}$. Then, $C$ is a smooth rational curve by Lemma 2.5.

Case I: $\left|C+\mathcal{F}+K_{S^{\prime}}\right| \neq \emptyset$.

By Lemma 2.6 and Claim, $S$ contains 3 rational double points and 1 more cyclic singularity. By Lemma $2.6, C$ is a (0)-curve or a $(-1)$-curve.

Case I-1: $C$ is a $(-1)$-curve.

Consider the case (2) of Lemma 2.6. Blowing up the intersection point of $C$ and $\mathcal{F}$, and then contracting the proper transform of $C$ and all irreducible components of $\mathcal{F}$, we obtain a rational $\mathbb{Q}$-homology projective plane $T$ with 6 quotient singularities which is a contradiction to Corollary 2.3.

Consider the case (1) or the case (3) of Lemma 2.6. Choose any intersection point of $C$ and $\mathcal{F}$. Blowing up the chosen point, and then contracting the proper transform of $C$ and all irreducible components of $\mathcal{F}$, we obtain a $\mathbb{Q}$-homology projective plane $T$ with $e_{\text {orb }}(T)<e_{\text {orb }}(S)=0$ which is a contradiction by Theorem 2.1.

Case I-2: $C$ is a (0)-curve.

The argument is similar to the previous case.

Consider the case (2) of Lemma 2.6. Blowing up the intersection point of $C$ and $\mathcal{F}$ and then blowing up again the intersection point of the two $(-1)$-curves before contracting the proper transform of $C$ and all irreducible components of $\mathcal{F}$ and a $(-2)$-curve, we obtain a rational $\mathbb{Q}$-homology projective plane $T$ with 5 quotient singularities which is a contradiction to Corollary 2.3. 
Consider the case (1) or the case (3) of Lemma 2.6. Choose any intersection point of $C$ and $\mathcal{F}$. Blowing up the chosen point, and then blowing up again the intersection point of the two $(-1)$-curves before contracting the proper transform of $C$ and all irreducible components of $\mathcal{F}$ and a $(-2)$-curve, we obtain a $\mathbb{Q}$-homology projective plane $T$ with $e_{\text {orb }}(T)<e_{\text {orb }}(S)=0$ which is a contradiction by Theorem 2.1 .

Case II: $\left|C+\mathcal{F}+K_{S^{\prime}}\right|=\emptyset$.

By Lemma 2.7, the minimal curve $C$ is a $(-1)$-curve. Moreover, $C . \mathcal{F}=1,2$, or 3 by Lemma 2.9. Let $F_{1}, F_{2}, \ldots, F_{r}$ be the irreducible components of $\mathcal{F}$ with $C . \mathcal{F}_{i}=1$ for $i=1,2, \ldots, r$ where $r=C . \mathcal{F}$. We may assume that $-F_{1}^{2} \leq$ $-F_{2}^{2} \leq \cdots \leq-F_{r}^{2}$ possibly by rearranging the index. We denote by $\mathcal{F}_{i}$ the connected component of $\mathcal{F}$ containing $F_{i}$ for $i=1,2, \ldots, r$. By Lemma $2.8, \mathcal{F}_{i}$ and $\mathcal{F}_{j}$ are disjoint if $i \neq j$.

Case II-1: The case $C . \mathcal{F}=1$. Assume that $\mathcal{F}_{1}$ is a linear chain. Blowing up the intersection point of $C$ and $F_{1}$, and then contracting the proper transform of $C$ and all irreducible components of $\mathcal{F}$, we obtain a rational $\mathbb{Q}$-homology projective plane $T$ with 5 singular points, which is a contradiction by Corollary 2.3 .

Assume that $\mathcal{F}_{1}$ is a fork. In our case, the noncyclic singularity $p$ is of type one of $D_{4}, D_{5}, D_{7}, D_{8}, E_{6},\langle 2 ; 2,1 ; 2,1 ; 3,1\rangle,\langle 3 ; 2,1 ; 2,1 ; 2,1\rangle$ by Table 2 . In any case, blowing up the intersection point of $C$ and $F_{1}$, and then contracting the proper transform of $C$ and all irreducible components of $\mathcal{F}$, we obtain a rational $\mathbb{Q}$-homology projective plane $T$ with at least 5 quotient singular points, which is a contradiction by Corollary 2.3.

Case II-2: The case $C . \mathcal{F} \geq 2$ and $F_{1}^{2}=F_{2}^{2}=-2$.

Now we consider the case $C . \mathcal{F} \geq 2$. Assume that $F_{1}^{2}=F_{2}^{2}=-2$. Then, by Lemma 2.11, $S$ contains at least two rational double points of type $A_{1}$. By the definition of the orbifold Euler characteristic, we see that $e_{\text {orb }}(S)>0$, which is a contradiction.

Case II-3: The case C.F $=2$.

By [12, Lemma 1.4], we may assume that $F_{1}^{2}=-2$. Let $F_{2}^{2}=-n$ for some integer $n$. By Case II-2, only the case $n \geq 3$ is remained.

Case II-3a: The case where $C$ meets only the linear chains in $\mathcal{F}$.

If $F_{1}$ is a tip, then blowing up the intersection point of $C$ and $F_{2}$, and then contracting the proper transform of $C$ and all irreducible components of $\mathcal{F}$, we obtain a $\mathbb{Q}$-homology projective plane $T$ with $e_{\text {orb }}(T)<e_{\text {orb }}(S)=0$ which is a contradiction by Theorem 2.1. Now we may assume that both $F_{1}$ and $F_{2}$ are not tips. Contracting $C$, and then contracting all images of irreducible components of $\mathcal{F}$ except $F_{1}$, we obtain a rational $\mathbb{Q}$-homology projective plane $T$ with 6 quotient singularities, which is a contradiction by Corollary 2.3.

Thus, we may assume that $C$ meets a fork in $\mathcal{F}$.

Case II-3b: The case where $C$ meets two forks in $\mathcal{F}$. 
Since $S$ admits at least 2 noncyclic singularities, only the case $(2,3,12,12)$ on the list ORDERS is possible and the 2 noncyclic singularities are of type $D_{5} \oplus D_{5}$. By assumption, $F_{1}^{2}=F_{2}^{2}=-2$. This case was already excluded in Case II-2.

Case II-3c: The case where $C$ does not meet a (-2)-curve in a fork.

In this case, $F_{2}^{2} \leq-3$. By looking at Table 2 , we see that $F_{2}^{2}=-3$. Only the 4-tuple $(2,3,8,24)$ on the list NONCYC satisfies this. Now, by looking at Table 1 , we see that $F_{1}$ is a tip of a linear chain. Blowing up the intersection point of $C$ and $F_{2}$, and then contracting the proper transform of $C$ and all irreducible components of $\mathcal{F}$, we obtain a $\mathbb{Q}$-homology projective plane $T$ with $e_{\text {orb }}(T)<e_{\text {orb }}(S)=0$, which is a contradiction to Theorem 2.3.

Case II-3d: The case where $C$ meets a (-2)-curve in a fork.

In this case, we may assume that $F_{1}$ is an irreducible component of a fork $\mathcal{F}_{1}$ with $F_{1}^{2}=-2$ and $F_{2}$ is an irreducible component of a linear chain $\mathcal{F}_{2}$ with $F_{2}^{2}=-n \leq-3$.

Claim that $F_{1}$ is a tip of $\mathcal{F}_{1}$. Suppose that $F_{1}$ is not a tip. Contracting $C$, and then contracting all images of irreducible components of $\mathcal{F}$ except $F_{1}$, we obtain a rational $\mathbb{Q}$-homology projective plane $T$ with at least 5 quotient singularities, which is a contradiction by Corollary 2.3 .

Assume that $F_{2}$ is also a tip. Consider, first, the case where $F_{1}$ meets the central component of the fork $\mathcal{F}_{1}$. First, we contract the minimal curve $C$. Second, we blow up the intersection point of the image $\bar{F}_{1}$ of $F_{1}$ and the central component $F_{C}$. Now, blowing up the intersection point of the exceptional divisor and the proper transform of $F_{C}$ sufficiently many times, and then contracting the proper transform of $C$ and all irreducible components of $\mathcal{F}$ and all the exceptional curves except the $(-1)$-curve, we obtain a $\mathbb{Q}$-homology projective plane $T$ with quotient singularities with $e_{\text {orb }}(T)<e_{\text {orb }}(S)=0$, which is a contradiction by Theorem 2.1 .

Now we consider the case where $F_{1}$ does not meet the central component of the fork $\mathcal{F}_{1}$. By Table 2 , we see that $\mathcal{F}_{1}$ is a minimal resolution of the singular point of type $D_{4}, D_{5}, D_{7}, D_{8}$, or $E_{6}$. Blowing up the intersection point of $C$ and $F_{1}$, and then contracting the proper transform of $C$ and all irreducible components of $\mathcal{F}$, we obtain a $\mathbb{Q}$-homology projective plane $T$ with quotient singularities with $e_{\text {orb }}(T)<e_{\text {orb }}(S)=0$, which is a contradiction.

Assume that $F_{2}$ is not a tip. First assume that the dual graph of the fork $\mathcal{F}_{1}$ is not $E_{6}$. Then, the fork $\mathcal{F}_{1}$ is a minimal resolution of one of the remaining six singularities listed in Table 2. In any case, blowing up the intersection point of $C$ and $F_{2}$, and then contracting the proper transform of $C$ and all irreducible components of $\mathcal{F}$, we obtain a $\mathbb{Q}$-homology projective plane $T$ with $e_{\text {orb }}(T)<e_{\text {orb }}(S)=0$, which is a contradiction to Theorem 2.3.

Now assume that the fork $\mathcal{F}_{1}$ is a resolution of the singular point of type $E_{6}$. So, only $(2,3,8,24)$ on the list NONCYC is possible. Note that since $F_{2}$ is not a tip by assumption, the singular point corresponding to $\mathcal{F}_{2}$ has a 
local fundamental group of order 8. If $F_{1}$ does not meet the central component of the fork $\mathcal{F}_{1}$, then we can derive a similar contradiction as above. If $F_{1}$ meets the central component of the fork $\mathcal{F}_{1}$, then contract the minimal curve $C$. Now, contracting all the images of $\mathcal{F}$ except $F_{1}$, we obtain a rational $\mathbb{Q}$ homology projective plane with 5 quotient singularities, which is a contradiction by Corollary 2.3.

Case II-4: The case $C . \mathcal{F}=3$.

Assume that $C . \mathcal{F}=3$. By Lemma 2.4 and Case II-2, we may assume that

$$
\left(-F_{1}^{2},-F_{2}^{2},-F_{3}^{2}\right)=(2,3, n)
$$

for some integer $n=3,4$ or 5 . Consider the divisor $G:=2 C+F_{1}+F_{2}+F_{3}+K_{S^{\prime}}$.

If $G \sim 0$, then each $\mathcal{F}_{i}$ consists of only one connected component that is $F_{i}$ by Lemma 2.12(1). By Lemma 2.4, we may assume that the triple of the orders of the local fundamental groups of the singular points $p_{1}, p_{2}$ and $p_{3}$ is one of $(2,3,3),(2,3,4)$, or $(2,3,5)$. It is easy to see that the 4 -tuple $\left(q_{1}, q_{2}, q_{3}, q_{4}\right)$ where $\left(q_{1}, q_{2}, q_{3}\right)$ is one of the above three triples is not on the list ORDERS.

Now, consider the case $G \sim \Gamma$ for some $(-1)$-curve $\Gamma$. Then, we may assume that $\mathcal{F}_{i}$ is a linear chain with $F_{i}$ as a tip by Lemma $2.12(2)(\mathrm{d})$. By looking at Table 1 , we see that, by Lemma $2.12(2)(\mathrm{b}-\mathrm{c}), F_{2}$ is one of $[3],[3,2],[3,2,2]$, $\left[3,2^{6}\right]$. We consider each of the three cases in Lemma 2.12.

Case II-4a: The case $\left(-F_{1}^{2},-F_{2}^{2},-F_{3}^{2}\right)=(2,3,5)$.

By looking at Table 1 , we see that $\mathcal{F}_{3}$ is one of $[5],[5,2]$, and $[5,5]$. The last case is excluded by Lemma $2.12(2)(\mathrm{c})$. Now, it is easy to see that only the following three cases on the list ORDERS satisfy this property.

- $(2,3,9,18),(2,4,5,20),(2,5,5,10)$

By Lemma 2.12(2)(a), the total number $L$ of irreducible components of $\mathcal{F}$ is exactly 12 .

Consider the case $(2,3,9,18)$. We see that $p_{1}$ is of type $A_{1}, p_{2}$ is of type [3] and $p_{3}$ is of type $[5,2]$. Thus, the number of irreducible component of $\mathcal{F}_{4}$ must be 8 , which is impossible by Table 1 and Table 2 .

Consider the case $(2,4,5,20)$. We see that $p_{1}$ is of type $A_{1}, p_{2}$ is of type either [4] or $A_{3}, p_{3}$ is of type [5] and $p_{4}$ is one of the types [7,3], [3, $\left.2^{3}, 3\right]$, $\left[2,3,2^{5}\right]$. Since $L=12$, only the combination $A_{1} \oplus A_{3} \oplus[5] \oplus\left[2,3,2^{5}\right]$ is possible. In this case, it is easy to see that, by [4, Lemma 3.6], $K_{S}^{2}=-\frac{5}{3}$, which is a contradiction.

Consider the case $(2,5,5,10)$. We may assume that $p_{1}$ is of type $A_{1}, p_{2}$ is of type [3,2], $p_{3}$ is of type [5] and $p_{4}$ is one of the types [10], [4,2,2], $A_{9}$. This is impossible since $L=12$.

Case II-4b: The case $\left(-F_{1}^{2},-F_{2}^{2},-F_{3}^{2}\right)=(2,3,4)$.

By Table 1 and Lemma $2.12(2)(\mathrm{b}-\mathrm{c})$, we see that $\mathcal{F}_{2}$ is one of [3], [3,2], $[3,2,2],\left[3,2^{3}\right],\left[3,2^{6}\right]$ and $\mathcal{F}_{3}$ is one of $[4],[4,2],[4,2,2]$. It is easy to see that the following six cases on the list ORDERS satisfy this property.

- $(2,3,7,42),(2,3,10,15),(2,4,5,20),(2,5,5,10),(3,3,4,12),(3,4,4,6)$ 
By Lemma 2.12(2)(a), $L=11$.

Consider the case $(2,3,7,42)$. We see that $p_{1}$ is of type $A_{1}, p_{2}$ is of type [3], $p_{3}$ is of type [4,2]. Since $L=11, p_{4}$ is of type $D_{7}$ or $\left[3,2^{3}, 3,2,2\right]$. In the first case, $K_{S}^{2}=-\frac{11}{21}$, which is a contradiction. The latter case is excluded by Lemma 2.12(2)(c).

Consider the case $(2,3,10,15)$. We see that $p_{1}$ is of type $A_{1}, p_{2}$ is of type [3], $p_{3}$ is of type $[4,2,2]$. Since $L=11, \Delta_{4}$ consists of 6 irreducible component, which is a contradiction by Table 1 .

Consider the case $(2,4,5,20)$. We see that $p_{1}$ is of type $A_{1}, p_{2}$ is of type [4], $p_{3}$ is of type $[3,2]$. Since $L=11, \Delta_{4}$ consists of 7 irreducible component, hence $\Delta_{4}=D_{7}$. In this case, $K_{S}^{2}=-\frac{3}{5}$, which is a contradiction.

Consider the case $(2,5,5,10)$. We see that $p_{1}$ is of type $A_{1}, p_{2}$ is of type $[3,2]$, $p_{4}$ is of type $[4,2,2]$. Since $L=11, \Delta_{3}$ consists of 5 irreducible component, which is a contradiction by Table 1 .

Consider the case $(3,4,4,6)$. We see that $p_{1}$ is of type [3], $p_{2}$ is of type $A_{3}$, $p_{4}$ is of type [4]. Since $L=11, \Delta_{3}$ consists of 6 irreducible component, which is a contradiction by Table 1 .

Consider the case $(3,3,4,12)$. We see that $p_{1}$ is of type $A_{2}, p_{2}$ is of type [3], $p_{4}$ is of type [4]. Since $L=11, \Delta_{3}$ consists of 7 irreducible component, which is a contradiction by Table 1 .

Case II-4c: The case $\left(-F_{1}^{2},-F_{2}^{2},-F_{3}^{2}\right)=(2,3,3)$.

By Table 1 and Lemma $2.12(2)(\mathrm{b}-\mathrm{c})$, we see that each of $\mathcal{F}_{2}$ and $\mathcal{F}_{3}$ is one of $[3],[3,2],[3,2,2],\left[3,2^{3}\right],\left[3,2^{6}\right]$. It is easy to see that the following six cases on the list ORDERS satisfy this property.

- $(2,3,7,42),(2,3,9,18),(2,3,10,15),(2,5,5,10),(3,3,4,12),(3,3,6,6)$.

By Lemma 2.12(2)(a), $L=10$.

Consider the case $(2,3,7,42)$. We see that $p_{1}$ is of type $A_{1}, p_{2}$ is of type [3], $p_{3}$ is of type $[3,2,2]$. Since $L=10, \Delta_{4}$ consists of 5 irreducible component, hence $\Delta_{4}=[2,2,6,2,2]$ by Table 1 , which is a contradiction by Lemma $2.12(2)(\mathrm{b})$.

Consider the case $(2,3,9,18)$. We see that $p_{1}$ is of type $A_{1}, p_{2}$ is of type [3], $p_{3}$ is of type $[3,2,2,2]$. Since $L=10, \Delta_{4}$ consists of 4 irreducible component, hence $\Delta_{4}=[3,3,2,2]$, which is a contradiction by Lemma $2.12(2)(\mathrm{c})$.

Consider the case $(2,3,10,15)$. We see that $p_{1}$ is of type $A_{1}, p_{2}$ is of type [3], $p_{4}$ is of type $\left[3,2^{6}\right]$. Since $L=10, \Delta_{3}$ consists of 1 irreducible component, hence $\Delta_{4}=[15]$, which is a contradiction by Lemma $2.12(2)(\mathrm{b})$.

Consider the case $(2,5,5,10)$. We see that $p_{1}$ is of type $A_{1}, p_{2}$ is of type $[3,2], p_{4}$ is of type $[3,2]$. Since $L=10, \Delta_{3}$ consists of 5 irreducible component, which is impossible.

Consider the case $(3,3,4,12)$. We see that $p_{1}$ is of type [3], $p_{2}$ is of type [3]. By Lemma 2.12(2)(b) and Table 1, $p_{3}$ is a rational double point, hence $p_{3}$ is of type $A_{3}$. Since $L=10, \Delta_{4}$ consists of 5 irreducible component, hence $\Delta_{4}=D_{5}$. In this case, it is easy to see that $K_{S}^{2}=-\frac{1}{3}$, which is a contradiction. 
Consider the case $(3,3,6,6)$. We see that $p_{1}$ is of type [3], $p_{2}$ is of type [3]. By Lemma 2.12(2)(b), both $p_{3}$ and $p_{4}$ are rational double points, hence of type $A_{5}$. This is a contradiction since $L=10$.

This concludes the proof of Theorem 1.1.

\section{Examples}

In this section, we construct a family of examples of log del Pezzo surfaces of rank one with arbitrarily small orbifold Euler characteristic.

Consider three concurrent lines $L_{1}, L_{2}$, and $L_{3}$ in $\mathbb{P}^{2}$ and consider one more line $L_{0}$.

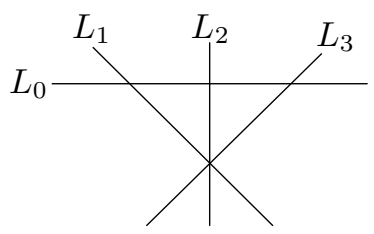

By blowing up the intersection point of the three lines $L_{1}, L_{2}$ and $L_{3}$, we obtain a Hirzebruch surface $\mathbb{F}_{1}$. The exceptional divisor $s$ is the minimal section and the proper transform $\tilde{L_{0}}$ of the line $L_{0}$ is a section of $\mathbb{F}_{1}$, and the proper transforms $\tilde{L_{1}}, \tilde{L_{2}}, \tilde{L_{3}}$ of the lines $L_{1}, L_{2}, L_{3}$ are fibers of $\mathbb{F}_{1}$. We will take 8 more blowups. First, blow up the three intersection points of $\tilde{L}_{0}$ and the three fibers $\tilde{L_{1}}, \tilde{L_{2}}, \tilde{L_{3}}$. Second, blow up the three intersection points of the exceptional divisors and the proper transforms $L_{1}^{2}, L_{2}^{2}, L_{3}^{2}$ of the three fibers $\tilde{L}_{1}, \tilde{L}_{2}, \tilde{L}_{3}$. Third, blow up the intersection point of the minimal section $s$ and $L_{3}^{2}$. Finally, blow up the intersection point of the exceptional divisor and the proper transform $L_{3}^{3}$ of $L_{3}^{2}$. Let $S^{\prime}$ be the surface we obtained and $L$ be the proper transform of $L_{3}^{3}$. The following picture describes the final configuration.

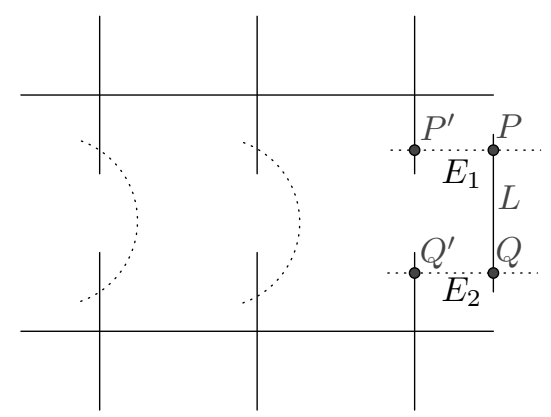

Note that $L$ is a $(-4)$-curve and, all the bold lines except $L$ are $(-2)$-curves and the dotted lines are all $(-1)$-curves.

Now, blow up the point $P(m-2)$ times, by which we mean the blowup of the point $P$ followed by the blowup of the intersection point of the exceptional 
divisor and the proper transform of $L$, and so on. Similarly, we blow up the point $Q(n-2)$-times. The surface we just obtained is called $S_{m+n}^{\prime}$. Then, we can find a $(-m-n)$-curve and $m+n+4(-2)$-curves forming a configuration $D_{m+2} \oplus D_{n+2}$ on $S_{m+n}^{\prime}$. By contracting those $m+n+5$ negative curves, we obtain a $\mathbb{Q}$-homology projective plane $S_{m, n}$ containing exactly three singular points of type $D_{m+2}, D_{n+2}$, and $[m+n]$. It follows that the orbifold Euler characteristic of $S_{m, n}$ is

$$
e_{\text {orb }}\left(S_{m, n}\right)=\frac{1}{m+n}+\frac{1}{4 m}+\frac{1}{4 n} .
$$

It is easy to verify that the anticanonical divisor $-K_{S_{m, n}}$ is ample using [5, Lemma 2.6]. Hence, $S_{m, n}$ is a log del Pezzo surface of rank one for each integer $m \geq 2$ and $n \geq 2$.

This completes the proof of Theorem 1.2.

Remark 4.1. By choosing $P^{\prime}$ or $Q^{\prime}$ rather than $P$ or $Q$ from $S^{\prime}$, one may obtain different families of examples of such surfaces.

\section{References}

[1] G. N. Belousov, Del Pezzo surfaces with log terminal singularities, Math. Notes 83 (2008), no. 1-2, 152-161.

[2] E. Brieskorn, Rationale Singularitäten komplexer Flächen, Invent. Math. 4 (1968), 336358.

[3] R. V. Gurjar and D. Q. Zhang, $\pi_{1}$ of smooth points of a log del Pezzo surface is finite. I, J. Math. Sci. Univ. Tokyo 1 (1994), no. 1, 137-180.

[4] D. Hwang and J. Keum, The maximum number of singular points on rational homology projective planes, J. Algebraic Geom. 20 (2011), no. 3, 495-523.

[5] _ Construction of singular rational surfaces of Picard number one with ample canonical divisor, Proc. Amer. Math. Soc. 140 (2012), no. 6, 1865-1879.

[6] _ Algebraic Montgomery-Yang Problem: the log del Pezzo surface case, to appear in J. Math. Soc. Jpn.

[7] S. Keel and J. McKernan, Rational curves on quasi-projective surfaces, Mem. Amer. Math. Soc. 140 (1999), no. 669, viii+153 pp.

[8] H. Kojima, Supplement to Normal del Pezzo surfaces of rank one with log canonical singularities by H. Kojima and T. Takahashi [J. Algebra 360 (2012), 53-70], J. Algebra 377 (2013), 312-316.

[9] H. Kojima and T. Takahashi, Notes on minimal compactifications of the affine plane, Ann. Mat. Pura. Appl. (4) 188 (2009), no. 1, 153-169.

[10] - Normal del Pezzo surfaces of rank one with log canonical singularities, J. Algebra 360 (2012), 53-70.

[11] Q. Ye, On Gorenstein log del Pezzo surfaces, Japan. J. Math. (N.S.) 28 (2002), no. 1, $87-136$.

[12] D. Q. Zhang, Logarithmic del Pezzo surfaces of rank one with contractible boundaries, Osaka J. Math. 25 (1988), no. 2, 461-497.

Department of Mathematics

AJou UnIVERSITY

SuwON 443-749, KoreA

E-mail address: dshwang@ajou.ac.kr 